\title{
Robust Penalty Adaptive Model Predictive Control (PAMPC) of Constrained, Underdamped, Non-collocated systems
}

4 Journal of Vibration and Control $(0: 1-15$

(CThe Author(s) 2010

Reprints and permission: sagepub.co.uk/journalsPermissions.nav DOI:

http:/mmsssagcpub.com (3)AGE

\author{
Abhishek Dutta; Clara Ionescu, Mia Loccufier and Robin De Keyser \\ Electrical Energy, Systems and Automation, Ghent University, \\ Sint-Pietersnieuwst. 41 Block B2, 9000 Gent, Belgium
}

\begin{abstract}
This paper investigates the control challenges posed by non-collocated mechatronic systems and motivates the need for a model-based control technique towards such systems. A novel way of online constraint handling by penalty adaptation (PAMPC) is proposed and shown to be of particular relevance towards robust control of underdamped, non-collocated systems by exploiting the structure of such systems. Further, a new tunneling approach is proposed for PAMPC to maintain feasibility under uncertainty. The PAMPC is shown to be optimal for control of a benchmark mass-spring-damper system, which poses all the mentioned challenges.
\end{abstract}

Keywords

constrained, predictive control, optimal, robust

\section{Introduction}

All actively controlled mechatronic systems are either collocated, or non-collocated. When the sensor measures at the same point where the actuation occurs, such systems are termed as collocated. It turns out that the dynamic characteristics of collocated systems are favourable for control system design. However, in real life, mechatronic applications generally are non-collocated and in addition underdamped which pose unique challenges for the control engineer. Some such cases include, bridges or flexible beams (Qiu et al., 2009) and production machines e.g. harvesters (Xie et al., 2013).

The flexihle beam model is often used to analyze several characteristics of underdamped, non-collocated systems and their control. In (Sokolov \& Babitsky, 2001), the cantilever beam model was used to study vibration suppression with non-collocated piezoelectric actuator and accelerometer. The proposed method of phase shifting was used to account for the non-collocation effects and was effective in suppressing the first two bending modes of the beam.

When controllers are designed for lightly damped structures, shifting or damping resonances is often the main concern. However, when it comes to non-collocated systems, anti-resonances should be considered as well. Further, an inaccurate model estimation may result in interchanging the order of poles and zeros. This, together with the presence of hard actuator constraints, could render the closed-loop unstable. Therefore, classical control techniques like pole-zero compensators

*Corresponding author |Wolfson College Cambridge]; e-mail: ad7L1@srcf.ucam.org 
with no systematic means of handling constraints can perform poorly given these characteristics of non-collocated systems (Preumont, 2011). Over the last three decades Model Predictive Control (MPC) has occupied the center stage in the control research community and had a tremendous impact on the process industry (H.Lee, 2011). Through the past decade there is a gradual shift to the mechatronic domain, mainly fueled by progress in fast nonlinear MPC algorithms. In a collocated setting, generalized predictive control has heen found to be suitable for damping the first vibration mode of a pinned-free beam model by (Richelot et al., 2004). (Brown et al., 2013) used Generalized predictive control for reducing the vibration of ground vehicles but have not considered constraints. The work carried by (Wills et al., 2008) highlighted the usefulness of MPC in handling constraints for active noise and vibration control. However, to our knowledge no results are reported on model based predictive control of non-collocated systems subjected to constraints.

In this paper, we present a novel online constraint management method, PAMPC and exploit the structure of underdamped non-collocated systems to tune the PAMPC in nominal as well as perturbed settings. Further, we present tunneling as a straightforward method to recover performance and feasibility under process disturbances as opposed to more conservative and computationally demanding approaches like min-max MPC (Maciejowski, 2002). The paper is structured as follows: Section 2 presents the characteristics of a non-collocated system by means of a mass-spring-damper (MSD) setup. A PID-controller is tuned to highlight the difficulties in the control of a non-collocated system. Section 3 gives a bricl on MPC and introduces the penalty adaptive constraint management system. The PAMPC design procedure for vibrating systems followed by robustness and feasibility analysis is detailed in section 4. A demonstration of PAMPC in nominal and perturbed settings on MSD setup is presented in section 5. Finally, the major conclusions of this research are presented in section 6 .

\section{Non-collocation: Characteristics and Control}

Non-collocation arises when the input force acts on the system at one point and the sensor measures the response at another. These are limitations posed by the design of the mechatronic systems. It may be seldom feasible to act and sense at the same point in reality.

Collocated systems, where the sensor and actuator are placed in the same position has the following property (Preumont, 2011 ): there is just one anti-resonance between two consecutive resonances. However, for non-collocated actuator-sensor systems, the above property is lost. It additionally poses the following problems:

1. As the sensor moves away from the actuator, the zeros migrate along the imaginary axis towards infinity and reappear from infinity on real axis.

2. If the resulting non-minimum phase zeros are within the system bandwidth, they can put severe restriction on the control system.

3. A pole-zero flipping might result due to modest variations in system parameters and can cause the corresponding branch in the root locus to become unstable!

As a direct consequence of the above, non-collocated control suffers from lack of robustness. We prove this over a mass-spring-damper set-up, that classical controller like PID lacks severely in terms of performance and stability.

\subsection{Mass-spring-damper setup}

The setup of Fig. 1(a) corresponds to a rectilinear electromechanical apparatus from ECP. The input of the plant is the voltage sent to the motor $u$ and the outputs of the plant are the mass displacements $\left(y_{1}\right.$ and $\left.y_{2}\right)$.

The electrical motor dynamics are fast compared to the mechanical dynamics, which means that the motor can be represented by a pure static gain:

$$
F(t)=K u(t)
$$




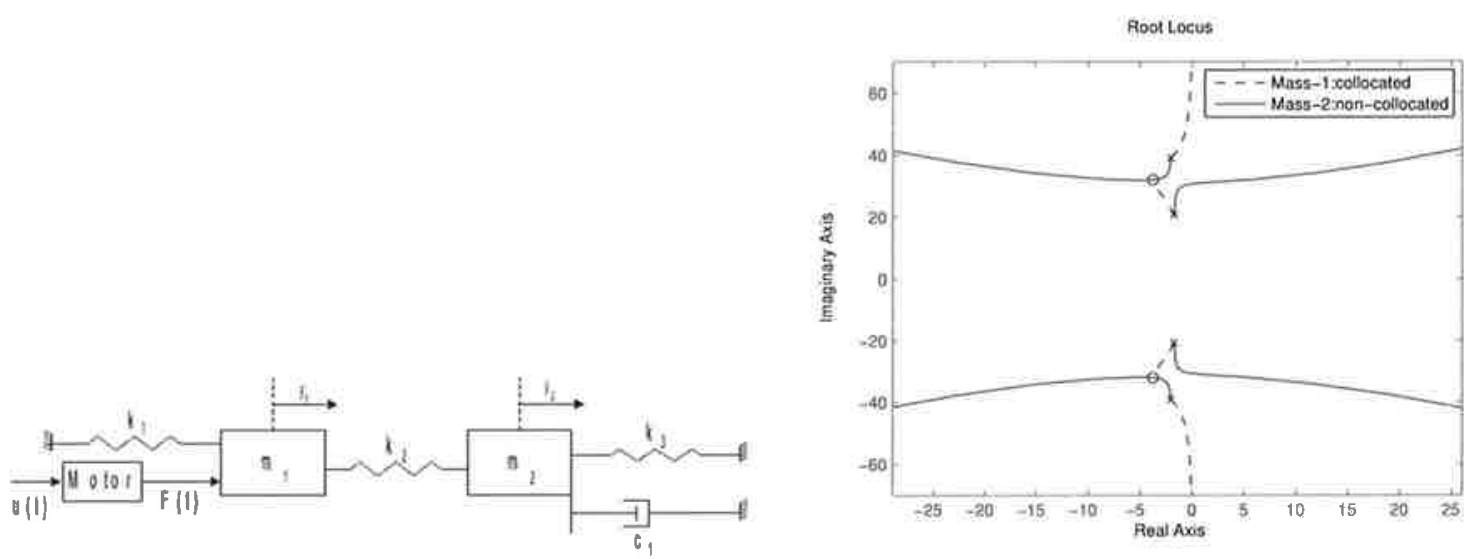

Fig. 1. (a): Mass spring damper setup, (b): Root locus of MSD demonstrating (non)collocation property

The parameters of the system are:

$$
\begin{aligned}
& m 1=1.7 \mathrm{~kg}, m 2=1.2 \mathrm{~kg}, k 1=k 2=800 \mathrm{~N} / \mathrm{m}, \\
& k 3=450 \mathrm{~N} / \mathrm{m}, c 1=9 \mathrm{~N} /(\mathrm{m} / \mathrm{s}), K=3.35 \mathrm{~N} / \mathrm{V}
\end{aligned}
$$

A mathematical representation of the system in Fig. l(a) is derived from the free body diagram and the application of Newton's Second Law of motion.

$$
\begin{aligned}
F(t) & =m_{1} \ddot{y}_{1}+k_{2}\left(y_{1}-y_{2}\right)+k_{1} y_{1} \\
0 & =m_{2} \ddot{y}_{2}+c_{1} \dot{y_{2}}+k_{3} y_{2}-k_{2}\left(y_{1}-y_{2}\right)
\end{aligned}
$$

Assuming zero initial conditions, the Laplace transform of (3) results in :

$$
\begin{aligned}
F(s) & =m_{1} s^{2} Y_{1}(s)+\left(k_{1}+k_{2}\right) Y_{1}(s)-k_{2} Y_{2}(s) \\
0 & =m_{2} s^{2} Y_{2}(s)+c_{1} s Y_{2}(s)+\left(k_{2}+k_{3}\right) Y_{2}(s)-k_{2} Y_{1}(s)
\end{aligned}
$$

Further algebraic manipulations lead to:

$$
\frac{Y_{1}}{F}=\frac{m_{2} s^{2}+c_{1} s+\left(k_{2}+k_{3}\right)}{\text { CharPoly }}, \quad \frac{Y_{2}}{F}=\frac{k_{2}}{\text { CharPoly }}
$$

or, including also model of the motor:

$$
\frac{Y_{1}}{U}=K \frac{m_{2} s^{2}+c_{1} s+\left(k_{2}+k_{3}\right)}{\text { CharPoly }}, \quad \frac{Y_{2}}{U}=K \frac{k_{2}}{\text { CharPoly }}
$$

where the characteristic polynomial CharPoly is given by:

$$
m_{1} m_{2} s^{4}+m_{1} c_{1} s^{3}+\left[m_{1}\left(k_{2}+k_{3}\right)+m_{2}\left(k_{1}+k_{2}\right)\right] s^{2}+c_{1}\left(k_{1}+k_{2}\right) s+k_{1} k_{2}+k_{2} k_{3}+k_{3} k_{1}
$$



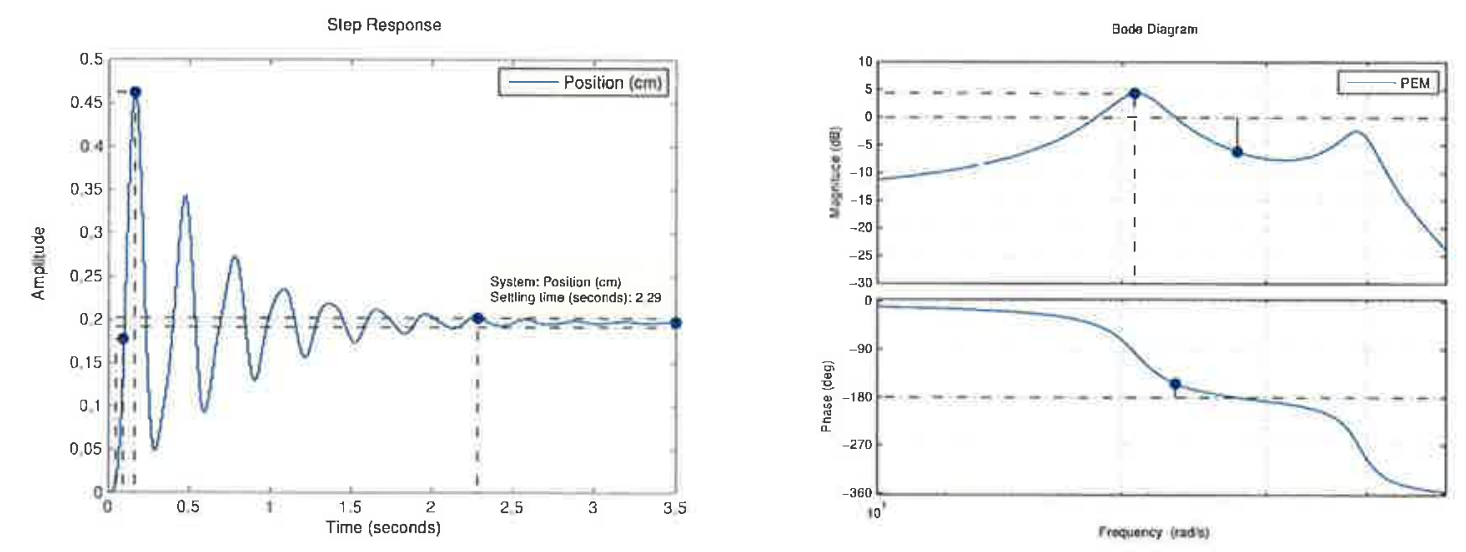

Fig. 2. (a): Mass-2 response to step input of $1 \mathrm{~V}$, (b): Mass-2 frequency response function

In the first casc, the position encoder measures the displacement of mass one $y_{1}$, at the same point as the input force from motor actuation, $u$, thus making the system collocated. In the second case, the displacement of mass two $y_{2}$ is measured at a different point than the input force $u$, thus making the system non-collocated.

The plot of Fig. 1(b) demonstrates some of the key distinguishing features of collocated and non-collocated systems. The first is the alternating poles and zeros near the imaginary axis. This is the case for collocated systems i.e. mass- 1 and does not exist for non-collocated systems like mass-2. Another principal feature is stability. For mass-1, the stable region is the negative real plane, and therefore this collocated system is and will be stable because the poles stay in the LH plane with increasing gain. However, for mass-2, since the imaginary zeros are no longer present, thus the system can very quickly become unstable as the poles travel to the positive RH plane. The step response of mass-2 is plotted in Fig. 2(a) which highlights the oscillatory response and long settling time.

A system identification using prediction error method is performed on the MSD system, and the corresponding frequency response function for the non-collocated case of mass-2 is plotted in Fig. 2(b). Multisine excitation signals covering the band of interest were used for the identification with $10 \mathrm{~ms}$ sampling time.

Recollect that, a feature of collocated systems like mass- 1 is the presence of an anti-resonance between two consecutive resonance frequencies. This means the phase always oscillates within $0^{\circ}$ and $-180^{\circ}$. Furthermore, the zeros of the collocated system are in fact the natural frequencies of the same system with the additional restraint at the collocated sensor and actuator. Since the anti-resonant frequencies are based on the actuator-sensor location, the mass- 2 bode plot of Fig. 2(b) depicts the absence of the anti-resonance between the same two resonant frequencies and thus there is no $180^{\circ}$ phase lead.

\subsection{PID control}

The objective is to control the position of the second mass which is a non-collocated scenario. A trivial but not at all suitable choice is PID control. The PID-controller possesses three tuning parameters: the proportional gain $K_{p}$, the integration time $T_{i}$ and the differentiation time $T_{d}$. Towards tuning, a relay feedback test with relay amplitude $r$ is applied to the process which makes the output oscillate around the set-point with a certain critical amplitude $A_{c}$ and critical period $T_{c}$. Consequently, the critical gain can be computed as $K_{c}=\frac{4 r}{\pi A_{c}}$. The experiment is performed on the mass-spring-damper and yields :

$$
r=0.5 \mathrm{~V}, T_{c}=0.25 \mathrm{~s}, A_{c}=0.35 \mathrm{~cm}
$$



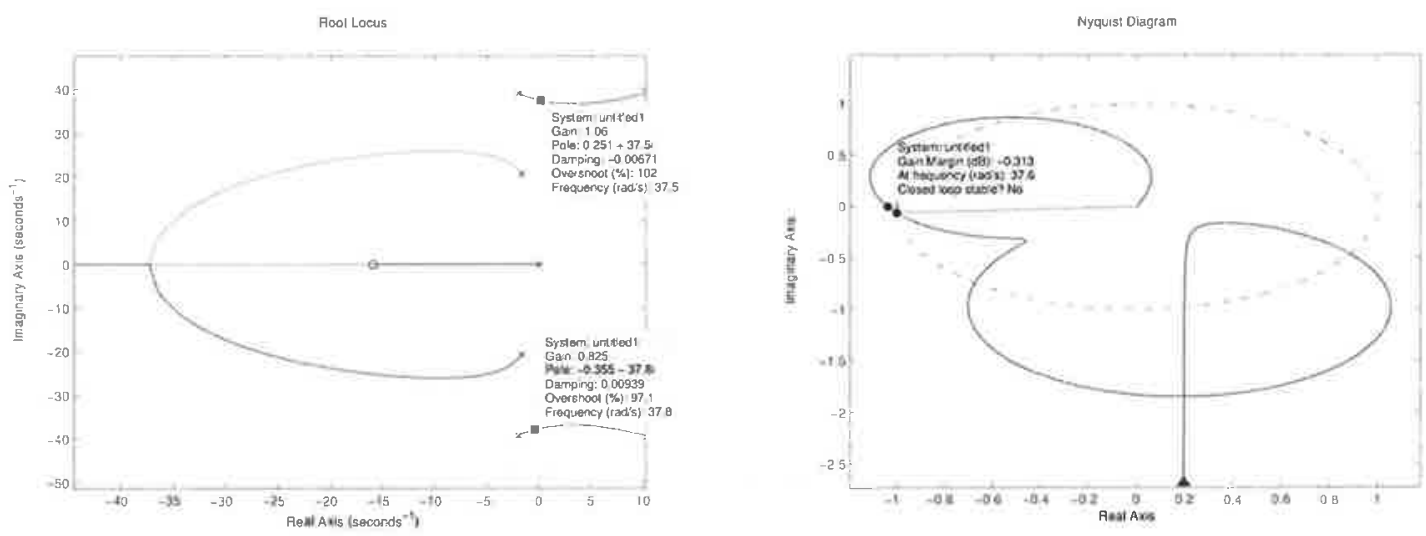

Fig. 3. (a): Auto-tuner root locus with a pair of overlapped zeros, (b): Nyquist of AH-autotuner open loop

Starting from (8), Aström and Hägglund have suggested several ways to compute the PID tuning parameters. We use a tuning method similar to Ziegler-Nichols (Astrom \& Wittenmark, 2011):

$$
K_{p}=0.6 K c ; \quad T_{d}=0.125 T c ; \quad T_{i}=4 T_{d}
$$

The resulting PID makes the closed loop unstable. This can be explained by the root locus analysis presented in Fig. $3(\mathrm{a})$.

In our case, the PID has a pole at origin and two overlapped real zeros. This clearly does not suffice to control a system with two pairs of underdamped poles. As marked on this plot, the closed loop corresponding to the tuning parameters (9) is clearly unstable, as the underdamped pole pair is already on the right half plane. This is a consequence of a non-collocated system with relative order 3, i.e. 3 zeros at infinity. The root locus of Fig. 3(a) has one asymptote along the negative real axis and two asymptotes at $\pm \frac{\pi}{3}$ with unstable branches.

This can be further explained with the Nyquist diagram of Fig. 3(b). The auto-tuning methods determine the critical frequency of the plant by the relay experiment and then enforce the open loop i.e. controller*plant to pass through the desired point on the complex plane at the plants critical frequency. However, in our special case with resonances, the open loop frequency response encircles the $(-1,0)$ point at a frequency greater then the critical frequency, which leads to the instability.

The poorly damped fourth order system gives an unstable closed loop when PID controllers are tuned with auto-tuners or at best oscillatory response with settling time larger than open loop with other tuning techniques (De Keyser et al., 2012). PIDs can neither control the oscillations, because of the poorly damped pole pair near the imaginary axis of the closed loop. A further inclusion of actuator limits can have disastrous consequences on the closed loop, as it is well known that clipping signals can make the system output unbounded (Camacho \& Bordons, 2003). Therefore, a more sophisticated model based control which cannot only counter the system dynamics but also deal with the constraints in a systematic manner, is deemed necessary.

\section{Constrained MPC by Penalty Adaptation}

MPC is a form of control in which the current control action is obtained by solving on-line, during each sampling period, a finite horizon open-loop optimal control problem (Maciejowski, 2002). This is done using the current state of the plant 
as the initial state for prediction of future states for which the optimization yields an optimal control sequence and the first control value in this sequence is applied to the plant (i.e. receding horizon). Next, we present a brief description of the Extended Prediction Self-Adaptive Control (EPSAC) and subsequently propose a novel semi-analytic formulation of handling constraints online.

\subsection{The EPSAC approach to MPC}

The process is modeled with $y(t), x(t), n(t)$ as process output, model output, disturbance respectively as (De Keyser, 2003):

$$
y(t)=x(t)+n(t)=\frac{q^{-d} B\left(q^{-1}\right)}{A\left(q^{-1}\right)} u(t-1)+\frac{C\left(q^{-1}\right)}{D\left(q^{-1}\right)} e(t)
$$

where $B / A$ represents the model dynamics with $d$ samples delay and $C / D$ is chosen to form the disturbance filter, with $e$ as white noise. The fundamental step is based on the prediction using the basic process model given by:

$$
y(t+k \mid t)=x(t+k \mid t)+n(t+k \mid t)
$$

where $y(t+k \mid t)$ is the prediction of process output $k$ steps in future computed at time $t$, over prediction horizon, based on prior measurements and postulated values of inputs. Prediction of model output $x(t+k \mid t)$ and of colored noise process $n(t+k \mid t)$ can be obtained by the recursion of process model and filtering techniques respectively. The future response can then be expressed as:

$$
y(t+k \mid t)=y_{\text {base }}(t+k \mid t)+y_{\text {optimize }}(t+k \mid t)
$$

The two contributing factors have the following origins:

- $y_{b a s e}(t+k \mid t)$ is the cumulative effect of past control inputs, base future control sequence $u_{\text {base }}(t+k \mid t)$ which is chosen a-priori and predicted disturbances.

- $y_{\text {optimize }}(t+k \mid t)$ is the discrete time convolution of the future control actions $\left\{\delta u(t \mid t) \ldots \delta u\left(t+N_{u}-1 \mid t\right)\right\}$ with impulse, step response coefficiencs of the system, where $\delta u(t+k \mid t)=u(t+k \mid t)-u_{\text {base }}(t+k \mid t)$.

The design parameter $N_{u}$ is the control horizon. The optimal control is then obtained by minimizing the following cost function:

$$
V\left(\delta C^{t}\right)=\Sigma_{k=N_{1}}^{N_{2}}[r(t+k \mid t)-y(t+k \mid t)]^{2}+\gamma
$$

where $r(t+k \mid t)$ is the desired reference trajectory (i.e. low pass filter). The prediction horizon is the interval from $N_{1}$ to $N_{2}$. The second cost term $\gamma$ can take any one of the following formulations:

$$
\gamma=\forall\left\{0, \lambda \Sigma_{k=0}^{N_{u}-1}[u(t+k \mid t)]^{2}, \lambda \Sigma_{k=0}^{N_{u}-1}[\delta u(t+k \mid t)]^{2}, \lambda \Sigma_{k=0}^{N_{u}-1}[\Delta u(t+k \mid t)]^{2}\right\}
$$

with $\lambda$ being the control penalty and control increment $\Delta u(t+k \mid t)=u(t+k \mid t)-u(t+k-1 \mid t)$.

\subsection{PAMPC: The Penalty Adaptation procedure}

We consider the following cost function formulation (Dutta et al., 2012):

$$
\begin{gathered}
V(\Delta U)=(R-Y)^{T}(R-Y)+\Delta U^{T} \Lambda \Delta U \\
\text { subject to }: \quad \Delta U \in U_{c}
\end{gathered}
$$


where $U_{c}$ is the set of convex constraints. In addition, $R, Y, \Delta U$ are now vectors of references, outputs, control increments respectively and $\Lambda$ a diagonal matrix of penalties. It is usual to define a first order reference trajectory over set-point with time-constant $\tau$.

The advantage of directly penalizing $\Delta U$ makes sense as it penalizes the high frequencies, which is of particular relevance for underdamped systems. It is well known that all forms of constraints i.e. on input, output, input rate can be accommodated in $U_{c}$. Now we have a quadratic programming (QP) problem which can be solved by interior-point or active-set based iterative optimizers (H.Lee, 2011). Note that, by doing so not only do we increase the computational burden but also loose the analytical solution to (15). Moreover a whole set of controller parameters need to be tuned.

The original contribution of this paper lies in re-formulating the entire constrained optimization problem to an equivalent unconstrained onc with adapted penaltics such that all the constraints are satisficd. Thus, as a first step all the controller parameters like horizons, etc., must be fixed beforehand based on the structure of the system (to be discussed in next section) and only then the penalty is adapted online to ensure optimal constraint satisfaction. We call this controller the PAMPC, the details follow.

In the second step, the controller is initialized with the unconstrained solution to (15), which is:

$$
\Delta U=\left(G^{T} G+\Lambda\right)^{-1} G^{T}(R-Y)
$$

i.e. the well known least squares solution with $G$, the step response matrix.

In the third step, we check for constraint violation. Let us say $v$ is the index of the constraint that is violated. Denote the error by $E=R-Y$. Now rewrite (16) as:

$$
\wedge . \Delta U=-G^{T} G \cdot \Delta U+G^{T} E
$$

The idea is to fix the violated constraint $\Delta u(t+v-1 \mid t)$ with its limit $u_{c}$ in the above system of equations and solve for the corresponding $\lambda_{c}$ together with the rest of the control inputs, thus maintaining the solvability of the system. Let us denote the $v^{\text {th }}$ column of matrix $G$ by $G_{v}$ and the matrix formed by rest of the columns other than $v$ by $\tilde{G}_{v}$. Similarly, let the vector $\tilde{\Delta U}$ venote all the elements except $v^{t h}$ and $\Delta u_{v}$ the $v^{t h}$ element. Finally $\Lambda_{v}$ denotes the $v^{t h}$ element of matrix $\Lambda$ and $\tilde{i}_{v}$ the matrix with $v^{\text {th }}$ row and column removed.

Collecting all the $\Delta u$ 's other than the one which is violated, we have:

$$
\tilde{\Lambda}_{v} \cdot \tilde{U}_{v}=-\tilde{G}_{v}^{T} \tilde{G}_{v} \cdot \tilde{\Delta U}_{v}-\Delta u_{v} \tilde{G}_{v}^{T} G_{v}+\tilde{G}_{v}^{T} E
$$

Thus, a solution to the above set of equations can be found as:

$$
\Delta \tilde{U}_{v}=\left(\tilde{G}_{v}^{T} \tilde{G}_{v}+\tilde{\Lambda}_{v}\right)^{-1}\left(-\Delta u_{v} \tilde{G}_{v}^{T} G_{v}+\tilde{G}_{v}^{T} E\right)
$$

The fourth step is to form the optimal control move vector $\Delta U$ by inserting the fixed value $u_{v}$ in the above computed control sequence $\tilde{\Delta U}{ }_{v}$. Now, we are in a position to compute the control penalty such that the active constraint $u_{v}$ is respected:

$$
\lambda_{v} \cdot \Delta u_{v}=-G_{v}^{T} G \cdot \Delta U+G_{v}^{T} E
$$

The steps three and four are repeated by sequentially checking for constraint violations in the future time steps until all constraints are salisfied. Once all constraints are salisfied, the absolute control applied to the plant is formed by: $u(t \mid t)^{*}=u(t-1)+\Delta u_{1}$. This forms the outer loop which runs within each sampling interval. In the next sampling time, this loop along with step two are repeated and so on. 
Theorem 1 Convergence of PAMPC control loop is always achieved.

Proof 1 Consider a compact constraint set with origin in its interior. The PAMPC algorithm makes $|\Delta u(t+v-1 \mid t)|$ smaller every time when it exceeds constraints by: fixing it to $\left|\Delta u_{v}\right|$. This in turn means that $\lambda_{v}$ increases with each iteration. Finally, infinite penalty on $\Delta u(t+v-1 \mid t)^{2}$ would mean $\Delta u(t+v-1 \mid t) \rightarrow 0, \forall v$, refer (16), which satisfies the stop criterion of PAMPC.

\section{Robust Design of PAMPC}

\subsection{Tuning of PAMPC by Structural Exploitation}

Here we present the choice of tuning parameters except penalty $\Lambda$ which are needed in the first step for PAMPC. Tuning of MPC controllers has drawn significant attention in the literature, however the vast majority of analytical tuning methods are applicable only when the constraints are inactive (Garriga \& Soroush, 2010). The rest of the tuning methods are trial and error iterative approaches (Darby \& Nikolaou, 2011). (Wang \& Boyd, 2010) point to the fact that a manifold increase in computation speed occurs if the structure of the problem at hand is exploited.

The computational complexity in each iteration scales with the square of control horizon i.e $O\left(N_{u}^{2}\right)$ for PAMPC and thus it is advisable to use short control horizons. However a control horizon of $N_{u}=1$, which results in mean-level control is not capable of optimal performance in high order systems which have multiple modes (D.W.Clarke et al., 1987). A time-optimal performance can definitely be guarantced by choosing the dead-beat settings for the unconstrained case.

Theorem 2 If $N_{2} \geq n_{A}+n_{B}+d+1, N_{1}=n_{B}+d+1, N_{u}=n_{A}+1$, then minimization of the cost function drives $y(t)$ in $n_{B}+d+1$ samples to the reference $\left(n_{A}, n_{B}\right.$ are the degrees of polynomials $A, B$ respectively).

Proof 2 Consider a regulation problem by minimization of the cost function (15) with zero penalty subject to equality constraints $\Delta u(t+k-1 \mid t)=0$ for $k>N_{2}$. This guarantees an unique solution since $N_{u}=N_{2}-N_{1}+1$ i.e the number of variables are same as number of equations. Further, after $N_{u}$ steps the controller must go to 0 ensuring output remains at 0 as well.

For the case with constraints, the control horizon $N_{u}$ and minimum prediction horizon $N_{1}$ remain the same as in dead-beat settings as these are strongly related to the structure of the plant. However, in the case of underdamped systems based on the rate of decay of the peak response, a prediction horizon of $N_{2}=2 * \omega_{s} / \omega_{b}$ i.e twice the sampling frequency over bandwidth frequency gives a good trade off between feasibility and optimality. This choice of $N_{u}<<N_{2}$ increases the stability of the closed loop, as this is equivalent to large terminal penalty. The time constant of the reference trajectory $\tau$ dictates the closed loop pole and thus must he fixed according to the desired speed of the closed loop. This leaves only the penalty term $\Lambda$ which should be initialized to a very small value $\leq 1 / N_{2}$ and is then adapted online by PAMPC. Note that, in general $A$ is fixed beforehand, and such a choice cannot be optimal under constraints.

\subsection{Robust Feasibility of PAMPC by Tunneling}

Robustness is delivered by PAMPC through an appropriate design of the disturbance filter. For well damped processes, it is common to choose $C / D=1 /\left(1-q^{-1}\right)$. However, for poorly damped systems, the 'integrator' disturbance filter gives oscillatory response.

Theorem 3 Part-1: If $A$ is a factor of $D$, then $A$ is no longer a factor of the closed-loop characteristic equation (CLCE). Part-2: Roots of $C$ appear as poles in the closed loop transfer function (CLTF). 

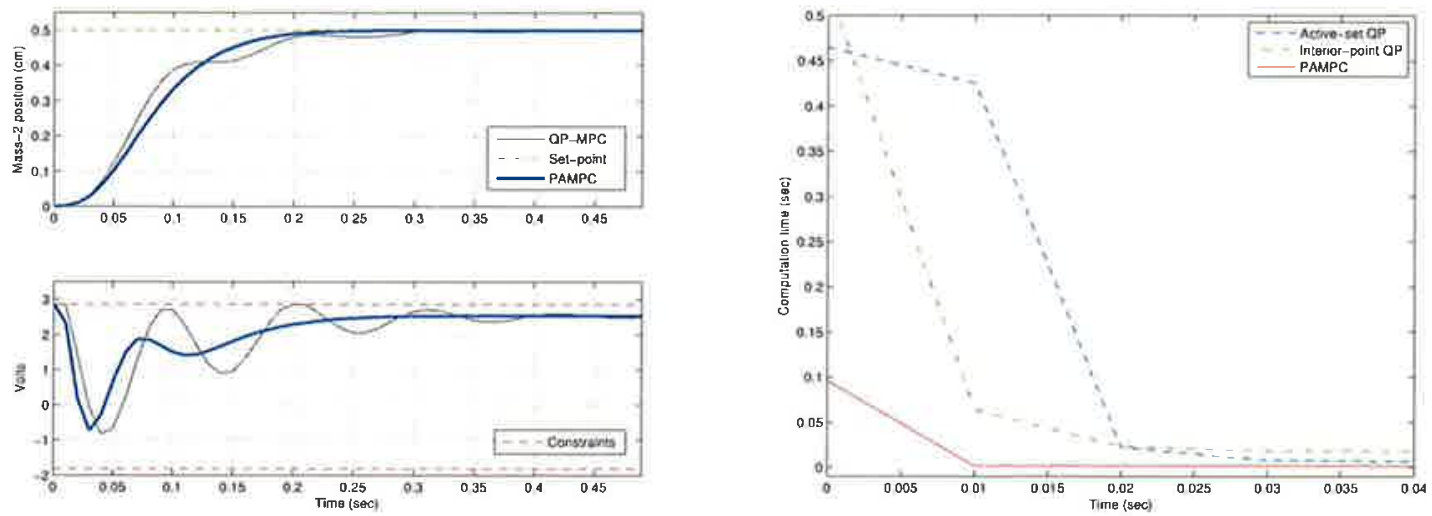

Fig. 4. (a): MSD control with PAMPC and QP, (b): Comparison of the computational costs

Proof 3 Part-1: The unconstrained MPC can be equivalently expressed in the following conventional 3-DOF form:

$$
I . u(t)=-J \cdot y(t)+K \cdot r(t)
$$

where $I, J, K$ are polynomials which can be computed by solving Diophantine equations (Dutta et al., 2014). It turns out that if $A$ is a factor of $D, A$ appears as a common factor of $I, J, K$ and thus cancels out of $C L C E$.

Part-2: The polynomial $C$ can be factored out of the LHS of the CLCE: $A . I+q^{-d-1} B . J=0$, obtained from (21), and hence the roots of $C$ appear as the poles of the CLTF.

Therefore, the first adaptation we make is to introduce the process denominator $A$ as an additional factor of $D$ in order to get a more stable response. The second step is to choose $C=\left(1-\rho \cdot q^{-1}\right)^{n_{A}}$ with $0 \leq \rho<1$ in order to ensure that the closed loop is stable. In general, higher values of parameter $\rho$ increases robustness but disturbances are rejected slower.

For constrained systems, however, feasibility should not be lost i.e. under possible acting disturbances, the controller output must lie within the constraints. As opposed to taking a conservative approach of computing the controller for the worst case scenario of disturbance sequence, we propose an online methodology to maintain feasibility by 'tunneling' i.e. creating tunnels through the input constraints. This approach is presented in the following two steps:

1. Compute and store the error at output as the difference between predicted model output and actual measured output i.e $n(t)$. From this, estimate the disturbance acting on the input: $u_{d}(t)=(A / B) . n(t)$. Note that the plant must be inverse stable. In case of noise, filtered measurements must be stored.

2. Predict future input disturbance based on: $u_{d}(t+k)=f\left(u_{d}(t+k-1), u_{d}(t+k-2), \ldots\right)$ where $k \in\left[0, N_{u}-1\right]$ and $f$ is a dynamic system learning kernel like neural network, the complexity of which depends on the complexity of the disturbance signal. Next, update the constraints based on the tunnel: $U_{t}=U_{c}-U_{d}$, where $U_{t}$ is the updated tunneled constraint trajectory and $U_{d}$ contains the predicted ' $u_{d}$ 's.

Theorem 4 Consider a PAMPC controller with disturbance filter designed for robustness against a class of disturbances. Robust feasibility can then be guaranteed if the predicted input disturbance sequence $U_{d}$ is subtracted from input constraints $U_{c}$ through the control horizon $N_{u}$.

Proof 4 Consider a robust PAMPC controller that computes an optimal, constraint admissible sequence $U^{*}$. Since, the optimization problem is solved for constraints $U_{c}$, any or all of the future control values can lie on the constraint. In that 

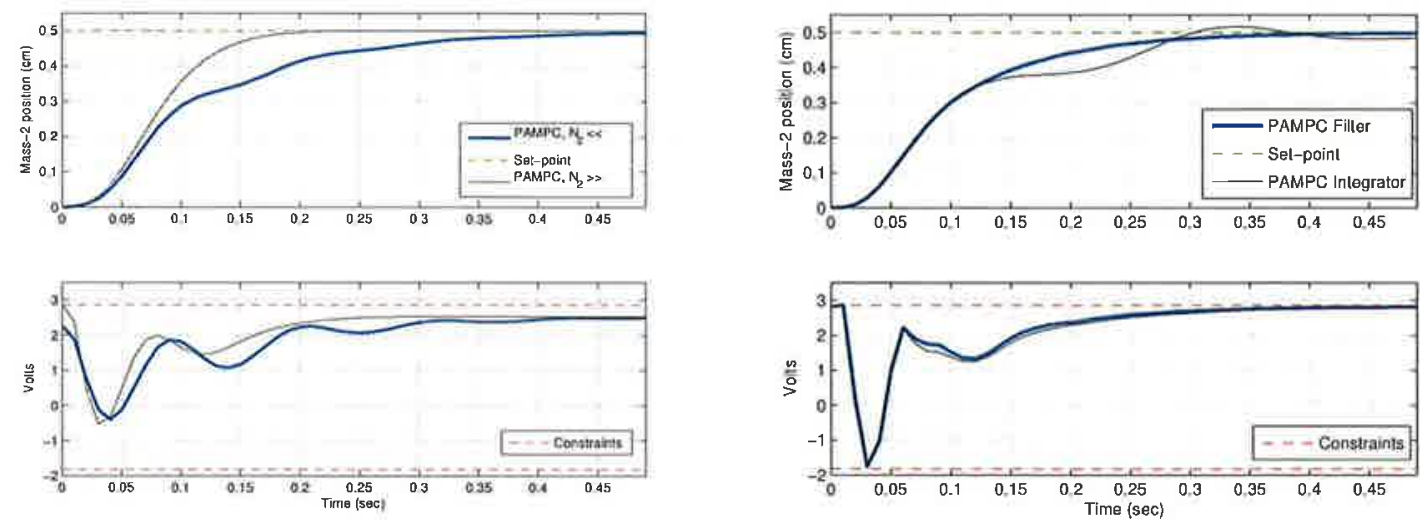

Fig. 5. (a): The result of suboptimal tuning, (b): Robust control under model uncertainty

case, the real control input to plant is: $U_{p}=U_{c}+U_{d}$, clearly violating the constraints. But, if the constraint set is tunneled to $U_{t}=U_{c}-U_{d}$, we have: $U_{p}=U_{t}+U_{d}=\left(U_{c}-U_{d}\right)+U_{d}=U_{c}$ which is feasible.

The above approach would guarantee that the robust control inputs remain constraint admissible even under process disturbances.

\section{PAMPC applied to position control of MSD}

MPC has been applied to vibrating systems, the most relevant would be the work by (Cairano et al., 2007) which deals with the predictive control of a mass spring damper system. However the study excludes analysis based on the structure of the system. The control system design based on the properties of underdamped non-collocated systems has been noted as a challenging problem in (Obrzut, 2009). Moreover, the majority of research in vibration control has focused on unconstrained systems (Beards, 1992). The PAMPC approach presented in this paper exploits the structure of the underdamped system in its design phase and manages the constraints online by finding optimal penalties. Let us demonstrate the efficiency of PAMPC on the MSD benchmark system.

Recall that, the objective is to control the position of mass-2 with an input voltage to the motor for fast response with minimum overshoot. This system is subjected to the following asymmetric input constraints:

$$
-1.82 \mathrm{~V} \leq u \leq+2.86 \mathrm{~V}
$$

The process model of (6) is used with the pole structure shown in Fig. 1(b), and the sampling time is same as before i.e. 10 ms. A discretization of the system yields $n_{B}=3, n_{A}=4, d=0$. We detail the PAMPC design procedure:

- $\quad$ Fix $N_{u}=n_{A}+1=5, N_{1}=n_{B}+d+1=4$.

- Compute rounded value of $N_{2}=2 * \omega_{s} / \omega_{b}=28$.

- Obtain a minimum positive integer $\tau$ which ensures no overshoot (through simulation), in this case $\tau=6$.

- Initialize $\Lambda=1 / N_{2}=0.03$.

With these settings the PAMPC controller is implemented on the MSD and the results are plotted in Fig. 4(a). As can be seen the settling time is within $0.23 \mathrm{~s}$ (10 times faster than open loop, see Fig. 2(a)) with no overshoot and the constraints are 

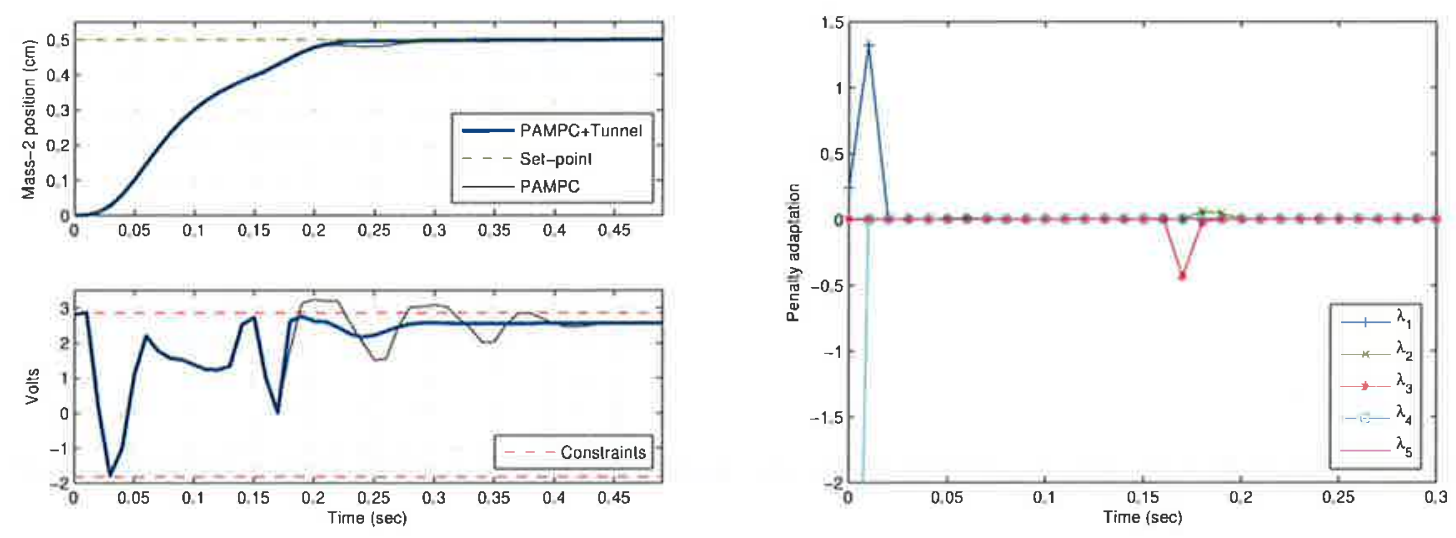

Fig. 6. (a): Robust feasibility under process disturbances, (b): Evolution of Penalty adaptations till convergence

respected as well. Notice that, in the beginning the maximum input constraint is active, thereby conforming the effective constraint management strategy by PAMPC.

For the sake of comparison, a MPC controller having more degrees of freedom is now designed with $N_{u}=14, N_{1}=$ $1, N_{2}=15, \Lambda=0.03, \tau=1$ and optimized by a QP solver instead of the penalty adaptation procedure. The constrained optimal solution in this case is plotted in the same Fig. 4(a) vis-a-vis PAMPC. PAMPC delivers much better performance for this underdamped non-collocated benchmark system. This is because the penalty matrix $\Lambda$ which has $N_{u}=5$ entries in its diagonal adapts considerably from initial value to deliver the required performance with constraint satisfaction. The $Q P$ based mean-level MPC however uses the conventional parameterization and can only guarantee constraint satisfaction, but has no means to find the correct penalty matrix and hence ends up with higher settling time and control effort. Computation time is a critical factor for the evaluation a control algorithm especially for fast systems, hence we compared the computation costs of active-set and interior-point QP solvers with PAMPC. The results are plotted in Fig. 4(b) for the first few iterations which are relevant due to active constraints. It is clear that PAMPC is at least 5 times faster than the QP solvers.

Further, we deliberately detune the prediction horizon only, once to half and then to double of its original value, to show the effectiveness of our suggested tuning procedure. It can be noted from Fig. 5(a) that a shorter $N_{2}=14$ induces oscillations, whereas higher $N_{2}=56$ does not lead to any improvement from $N_{2}=28$ of Fig. 4(a) but certainly increases the computation burden. Next, we consider the case where the model has $\pm 5 \%$ uncertainty in terms of the gain, the two natural frequencies and damping ratios. Under these settings, the PAMPC with the same parameters as above but now with the disturbance filter designed as: $C / D=1 /\left(A .\left(1-q^{-1}\right)\right)$ is considered. The results are plotted in Fig. 5(b) which shows the settling time is now just over $0.3 \mathrm{~s}$ and has zero overshoot. This is then compared to the one where a standard integrator filter is used. Notice, that in this case the oscillations persist in the controlled closed loop.

In a last test, an additive step disturbance equal to one third of the input range is introduced at $0.15 \mathrm{~s}$. In this scenario we keep the above tuning with the improved filter for PAMPC and add the tunneling mechanism from the previous section. The learning function here is just a constant with no memory; the results are illustrated in Fig. 6(a). The PAMPC controller maintains the nominal performance with no constraint violations. Notice that Fig. 6(b), the penalties are changed once again after $0.15 \mathrm{~s}$ to adapt to the step disturbance, before they converge again. This is compared to the controller without tunneling, and it can be clearly seen that this results in serious constraint violations. 


\section{Conclusions}

In this paper, the control challenges posed by constrained, underdamped, non-collocated mechatronic systems have been highlighted through a mass-spring-damper representative system. First, a novel predictive control strategy PAMPC is introduced which manages constraints by penalty adaptation and can be tuned by considering the structure of the system in its design phase. Second, an online constraint tunneling approach is presented towards robust feasibility of the PAMPC controller under process disturbances. Such a methodology has been demonstrated to achieve superior performance for the robust control of the MSD system.

\section{Acknowledgements}

Abhishek Dutta is supported within the framework of the LeCoPro project (grant nr. 80032) of the Institute for the Promotion of Innovation through Science and Technology in Flanders (IWT-Vlaanderen). C.M. Ionescu is a post doctoral researcher of the Flanders Research Foundation (FWO).

\section{References}

Astrom K. \& Wittenmark B. (2011). Computer-controlled Systems: Theory and Design. Dover Publications.

Beards C. (1992). Vibrations and control systems. E. Horwood.

Brown R., et al. (2013). 'Generalized predictive control algorithm of a simplified ground vehicle suspension system'. Journal of Vibration and Control 19(16):2372-2386.

Cairano S. D., et al. (2007). 'Model predictive control of magnetically actuated mass spring dampers for automotive applications'. International Journal of Control 80(11):1701-1716.

Camacho E. \& Bordons C. (2003). Model predictive control. Springer-Verlag.

Darby M. \& Nikolaou M. (2011). 'MPC: Current practice and challenges'. Control Engineering Practice, 20 pp. $328-342$.

De Keyser R. (2003). 'Model based predictive control for linear systems'. UNESCO Encyclopaedia of Life Support Systems http://www.eolss.net. Article contribution 6.43.16.1, Eolss Publishers Co Ltd, Oxford (2003) 35 pages.

De Keyser $R_{,}, \mathrm{Cl}$ a]. (2012). 'A specifications based PII) auloluner'. In Control Applications (CCA), 2012 IEEE International Conference on, pp. 1621-1626.

Dutta A., et al. (2014). 'Robust and two-level (nonlinear) predictive control of switched dynamical systems with unknown references for optimal wet-clutch engagement'. Proceedings of the Institution of Mechanical Engineers, Part I: Journal of Systems and Control Engineering 228(4):200-212.

Dutta A., et al. (2012). 'Switched predictive control design for optimal wet-clutch engagement'. In IFAC Workshop on Engine and Powertrain Control, Simulation and Modeling (ECOSM-2012), pp. 319-324.

D.W.Clarke, et al. (1987). 'Generalized predictive control-I. the basic algorithm'. Automatica pp. 137-148.

Garriga J. L. \& Soroush M. (2010). 'Model predictive control tuning methods: A review'. Industrial \& Engineering Chermistry Research $49(8): 3505-3515$.

H.Lee J. (2011). 'Model Predictive Control: Review of the Three Decades of Development'. International Journal of Control, Automation, and Systems pp. 415-424.

Maciejowski J. (2002). Predictive Control with Constraints. Prentice Hall.

Obrzut T. (2009). Non-Collocation Problems in Dynamics and Control of Mechanical Systems. Cleveland State University.

Preumont A. (2011). Vibration Control of Active Structures. Springer.

Qiu Z., et al. (2009). 'Active Vibration Control of a Flexible Beam Using a Non-Collocated Acceleration Sensor and Piezoelectric Patch Actuator'. Journal of Sound and Vibration, Vol. 326 pp. $438-455$.

Richelot J., et al. (2004). 'Active control of a clamped beam equipped with piezoelectric actuator and sensor using generalized predictive control'. In Industrial Electronics, 2004 IEEE International Symposium on, vol. 1, pp. 583-588. 
Sokolov I. \& Babitsky V. (2001). 'Phase control of self-sustained vibration'. Journal of sound and vibration 248(4):725-744.

Wang Y. \& Boyd S. (2010). 'Fast Model Predictive Control Using Online Optimization'. IEEE Trans. On Control Systems Tech., Vol.18, No.2 pp. 267-278.

Wills A., et al. (2008). 'Model predictive control applied to constraint handling in active noise and vibration control'. IEEE Transactions on Control Systems Technology, I6(l) pp. 3-12.

Xie Y., et al. (2013). 'Fundamental limits in combine harvester header height control', Journal of dynamic systems, measurement, and control 135(3): 1-8. 
